\title{
Wavelets and splines for modelling the earth's magnetic field
}

\author{
Carsten Mayer ${ }^{1, *}$ \\ ${ }^{1}$ University of Kaiserslautern, Geomathematics Group, P.O. Box 3049, D-67653 Kaiserslautern, Germany.
}

\begin{abstract}
At the present time there is an unprecedented opportunity to use satellite magnetometer data, as for example Oersted and CHAMP records, to improve our knowledge of the Earth's magnetic field and its sources. These measurements contain contributions from the main magnetic field of the Earth, the crustal field as well as contributions coming from different exterior (ionospheric and magnetospheric) sources. For the mathematical analysis of the data it is critical to have in hand appropriate tools which separate these sources and which are able to analyze the different contributions independently. We present in this talk an approach in terms of (spherical) scalar and vectorial scaling functions and wavelets to handle these problems. Examples include crustal field modelling and reconstruction of ionospheric currents.
\end{abstract}

Copyright line will be provided by the publisher

\section{Modelling the Earth's Magnetic Field}

In this article we are dealing with vectorial magnetic field data of the Earth measured by satellites. Thus, we first assume that the data is measured on a sphere $\Omega$ in the outer space of the Earth. For modelling the magnetic field we need certain systems of vector spherical harmonics on the sphere. The system we use in this article is denoted by $\left\{u_{n, k}^{(i)}\right\}$ (see [1]). Let $f \in l^{2}(\Omega)$, i.e. $f$ is a square integrable vector field on the unit sphere $\Omega$, be given, then we have (see [3])

$$
f=\sum_{n=0}^{\infty} \sum_{k=1}^{2 n+1}\left(f^{(1)}\right)^{\wedge}(n, k) u_{n, k}^{(1)}+\sum_{n=1}^{\infty} \sum_{k=1}^{2 n+1}\left(\left(f^{(2)}\right)^{\wedge}(n, k) u_{n, k}^{(2)}+\left(f^{(3)}\right)^{\wedge}(n, k) u_{n, k}^{(3)}\right),
$$

in the sense of the $l^{2}(\Omega)$-norm with the coefficients given by

$$
\left(f^{(1)}\right)^{\wedge}(n, k)=\left(u_{n, k}^{(1)}, f\right)_{l^{2}(\Omega)}, \quad\left(f^{(2)}\right)^{\wedge}(n, k)=\left(u_{n, k}^{(2)}, f\right)_{l^{2}(\Omega)}, \quad\left(f^{(3)}\right)^{\wedge}(n, k)=\left(u_{n, k}^{(3)}, f\right)_{l^{2}(\Omega)} .
$$

Using the construction of the system $\left\{u_{n, k}^{(i)}\right\}$ (see [1]), it can be shown that the first part represents the field resulting from sources inside the sphere $\Omega$, the second part is coming from sources outside $\Omega$ and the third part is the toroidal part of $f$ on $\Omega$. Using the system $\left\{u_{n, k}^{(i)}\right\}$ of vector spherical harmonics we next define vector scaling functions and wavelets. Let $\left\{\left(\phi_{J}^{(i)}\right)^{\wedge}(n)\right\}_{n \in \mathbb{N}}$ for $J \in \mathbb{Z}$ and $i \in\{1,2,3\}$, be a family of symbols, satisfying $\lim _{J \rightarrow \infty}\left(\phi_{J}^{(i)}\right)^{\wedge}(n)=1$ for all $n \in \mathbb{N}_{0}$ and $\left(\phi_{J}^{(i)}\right)^{\wedge}(n) \leq\left(\phi_{J+1}^{(i)}\right)^{\wedge}(n)$ for all $J \in \mathbb{Z}, n \in \mathbb{N}_{0}$. The vector scaling functions of type $i$ is, for $\xi, \eta \in \Omega$, defined by

$$
\phi_{J}^{(i)}(\xi, \eta)=\sum_{n=0}^{\infty} \sum_{k=1}^{2 n+1}\left(\phi_{J}^{(i)}\right)^{\wedge}(n) u_{n, k}^{(i)}(\xi) Y_{n, k}(\eta), \quad \xi, \eta \in \Omega .
$$

Let $f \in l^{2}(\Omega)$ and $F \in \mathrm{L}^{2}(\Omega)$, then the (decomposing) convolution of $\phi_{J}^{(i)}$ against $f$ and the (reconstructing) convolution of $\phi_{J}^{(i)}$ against $F$ are defined by

$$
\left(\phi_{J}^{(i)} * f\right)(\xi)=\int_{\Omega} \phi_{J}^{(i)}(\eta, \xi) \cdot f(\eta) d \omega(\eta), \quad\left(\phi_{J}^{(i)} \star F\right)(\xi)=\int_{\Omega} \phi_{J}^{(i)}(\xi, \eta) F(\eta) d \omega(\eta), \quad \xi \in \Omega . \quad \xi \in \Omega .
$$

The two types of convolutions satisfy a substantial property of Fourier analysis, namely that the convolution of two functions in spatial domain is equivalent to multiplication of the Fourier representations of the two functions in the spectral domain. Furthermore, it can be shown that, for $f \in l^{2}(\Omega)$ we have $\lim _{J \rightarrow \infty}\left\|\phi_{J}^{(i)} \star \phi_{J}^{(i)} * f-f^{(i)}\right\|_{l^{2}(\Omega)}=0$, where $f^{(i)}$ is the part of $f$ in the linear span of the system $\left\{u_{n, k}^{(i)}\right\}$. In the case $i=1$ this is an approximation formula for that part of $f$ which is generated by sources inside $\Omega$, if $i=2$ it is the part of $f$ which is generated by sources outside $\Omega$ and in the case $i=3$ it is an approximation of the toroidal part of $f$ which is generated by the radial projection of source fields (currents) crossing $\Omega$.

The main advantage of the above theorem results from a substantial property of the theory of kernel functions, i.e. the localization property. Scaling functions are known as very well space localizing functions, since they are clusters of a system of orthonormal functions in the corresponding Hilbert space. The mathematical background for the discrepancy between spatial and spectral localization is given by the uncertainty principle (see [2]). A graphical illustration of the uncertainty principle 
Table 1 A graphical illustration of the uncertainty principle.

\begin{tabular}{|c|c|c|}
\hline Ideal frequency localization & & No frequency localization \\
\hline No space localization & & Ideal space localization \\
\hline vector spherical harmonics, $u_{n, k}^{(i)}$ & vector kernels, $\phi_{J}^{(i)}$ & Dirac functional, $\delta_{\Omega}^{(i)}$ \\
\hline
\end{tabular}

and the construction of vector kernel functions is given in Table 1. By the space localizing property of scaling functions and wavelets we are able to separate a given field $f \in l^{2}(\Omega)$ locally with respect to its sources. In consequence, we do not need a global coverage of data. With data given only in a regional area of the unit sphere $\Omega$ we are able to decide which part of $f$ results from sources inside, outside or on the sphere.

To complete the theory of a multiscale decomposition of the identity, corresponding vector wavelets, a wavelet transform and certain filter operators can be defined. For more details the reader is referred to [3] and [4].

\section{Application to CHAMP Vector Magnetic Data}

In this section we apply the previously presented multiscale approach for separating given spherical vector fields with respect to their sources to a set of vector magnetic data of the geoscientific satellite mission CHAMP (see http://www.gfzpotsdam.de/pb1/op/champ/). The magnetic field data set we use has been made available by Stefan Maus from the GFZ Potsdam. For more information concerning the data pre-processing the reader ist referred to [4].

Figure 1 shows a multiscale decomposition of a CHAMP geomagnetic field data set over Africa (Bangui anomaly) using a type 1 Shannon vector scaling function $\left(\operatorname{symbol}\left(\phi_{J}^{(1)}\right)^{\wedge}(n)=1\right.$ for all $\left.n=0, \ldots, 2^{J}\right)$ at scale $J=7$. The left part shows the original data set after certain pre-processing steps (subtraction of the geomagnetic main field). The figure in the middle shows the filtered part using the type 1 Shannon vector scaling function. Due to the theory presented before, this is the part of the field which is generated by sources inside the satellite's orbital sphere. The right part shows the difference of the two other figures. In case of crustal geomagnetic field modelling the filtered data set is a enormous improvement since the difference of the two fields shows structures which are clearly not due to crustal sources. This shows, that the technique of space localizing calculations using vector scaling functions and wavelets is especially applicable for modelling and analyzing small spatial effects like the geomagnetic field of the Earth's crust.
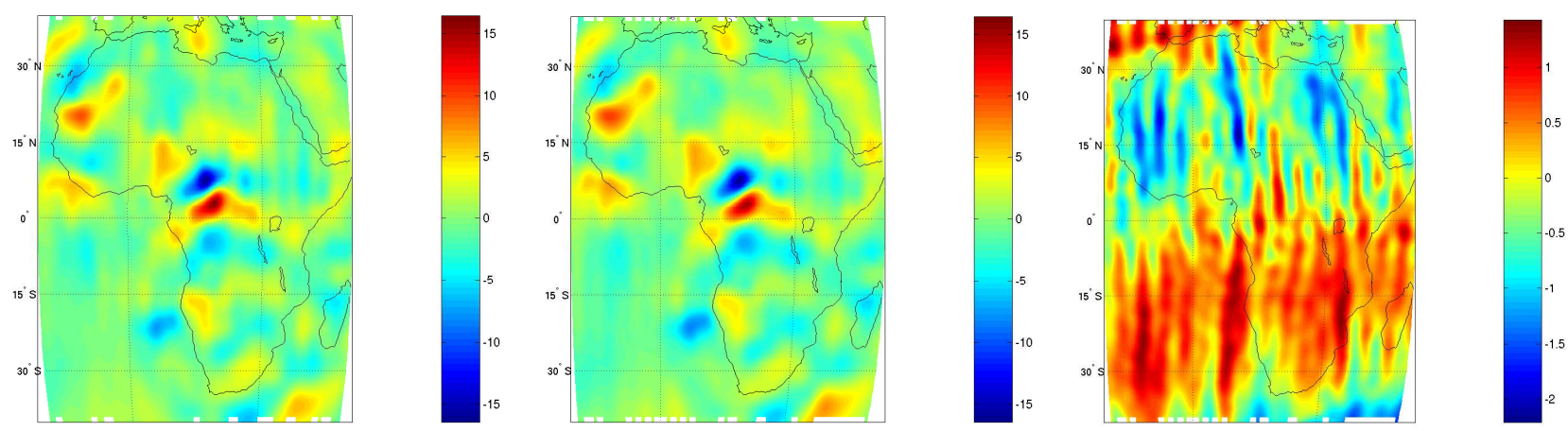

Fig. 1 Local multiscale decomposition of a CHAMP geomagnetic field data set over Africa (Bangui anomaly) using a type 1 Shannon vector scaling function at scale $J=7$. The left part shows the original data set, the figure in the middle shows the filtered part using the type 1 Shannon vector scaling function which is the part of the field generated by sources inside the satellite's orbital sphere. The right part shows the difference of the two other figures.

\section{References}

[1] G.E. Backus, R. Parker, and C. Constable, Foundations of Geomagnetism, (Cambridge University Press, Cambridge, 1996).

[2] W. Freeden, T. Gervens, and M. Schreiner, Constructive Approximation on the Sphere (With Applications to Geomathematics), (Oxford Science Publications, Clarendon, 1998).

[3] C. Mayer, Wavelet Decomposition of Spherical Vector Fields with Respect to Sources, Journal of Fourier Analysis and Applications, 12(4), 345-369, (2006).

[4] C. Mayer, T. Maier, Separating Inner and Outer Earth's Magnetic Field from CHAMP Satellite Measurements by Means of Vector Scaling Functions and Wavelets, Geophysical Journal International (GJI), 167(3), 1188-1203, (2006).

* Corresponding author E-mail: cmayer@mathematik.uni-kl.de, Phone: +49 (0)631 2054584 , Fax: +49 (0)631 2054736 